\title{
From network structure to network reorganization: implications for adult neurogenesis
}

\author{
Casey M Schneider-Mizell ${ }^{1,2}$, Jack M Parent ${ }^{3}$, Eshel Ben-Jacob ${ }^{4}$, \\ Michal R Zochowski ${ }^{1,5}$ and Leonard M Sander ${ }^{1}$ \\ ${ }^{1}$ Department of Physics, University of Michigan, Ann Arbor, MI 48109, USA \\ ${ }^{2}$ Institute for Neuroinformatics, University of Zurich and ETH Zurich, Zurich, Switzerland \\ ${ }^{3}$ Department of Neurology, University of Michigan, Ann Arbor, MI 48109, USA \\ ${ }^{4}$ School of Physics and Astronomy, Tel-Aviv University, Tel Aviv, Israel \\ ${ }^{5}$ Biophysics Program, University of Michigan, Ann Arbor, MI 48109, USA \\ E-mail: lsander@umich.edu
}

Received 20 July 2010

Accepted for publication 15 October 2010

Published 12 November 2010

Online at stacks.iop.org/PhysBio/7/046008

\begin{abstract}
Networks can be dynamical systems that undergo functional and structural reorganization. One example of such a process is adult hippocampal neurogenesis, in which new cells are continuously born and incorporate into the existing network of the dentate gyrus region of the hippocampus. Many of these introduced cells mature and become indistinguishable from established neurons, joining the existing network. Activity in the network environment is known to promote birth, survival and incorporation of new cells. However, after epileptogenic injury, changes to the connectivity structure around the neurogenic niche are known to correlate with aberrant neurogenesis. The possible role of network-level changes in the development of epilepsy is not well understood. In this paper, we use a computational model to investigate how the structural and functional outcomes of network reorganization, driven by addition of new cells during neurogenesis, depend on the original network structure. We find that there is a stable network topology that allows the network to incorporate new neurons in a manner that enhances activity of the persistently active region, but maintains global network properties. In networks having other connectivity structures, new cells can greatly alter the distribution of firing activity and destroy the initial activity patterns. We thus find that new cells are able to provide focused enhancement of network only for small-world networks with sufficient inhibition. Network-level deviations from this topology, such as those caused by epileptogenic injury, can set the network down a path that develops toward pathological dynamics and aberrant structural integration of new cells.
\end{abstract}

S Online supplementary data available from stacks.iop.org/PhysBio/7/046008/mmedia

\section{Introduction}

In adult hippocampal neurogenesis, a continuous population of new cells integrates into the existing active network of neurons of the dentate gyrus (DG). The process is thought to assist memory formation and storage by reinforcing precise activity patterns while leaving intact the large-scale structural and functional properties of the network [1, 41, 47]. This suggests that adult neurogenesis is optimized for the maintenance of functional activity during structural turnover. However, it is also known that epileptogenic injury causes structural changes to the network environment around new cells, dramatically altering existing connectivity patterns, and is associated with aberrant integration of new cells and possibly the development of epilepsy [34]. In this paper, we use a computational model to investigate the role of the existing, established network 

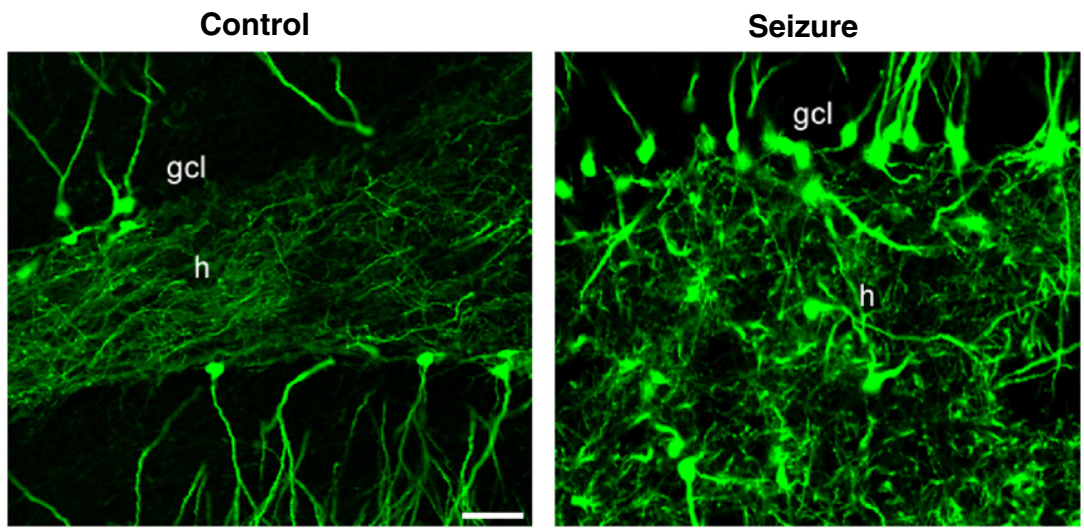

Figure 1. Coronal brain sections through the dorsal hippocampal dentate gyrus of adult rats immunostained for green fluorescent protein (GFP). A retrovirus carrying GFP was injected 24 days earlier, and 4 days after saline (left) or pilocarpine chemoconvulsant (right) treatment. The retroviral GFP specifically labeled dividing dentate granule cell (DGC) progenitors in the adult that have subsequently differentiated into DGCs. The control shows a normal pattern of DGC development, while the epileptic (seizure) rat shows markedly abnormal DGC development, with cell bodies and processes abnormally located and mis-oriented. gcl, granule cell layer; $\mathrm{h}$, hilus. Scale bar $=50 \mu \mathrm{m}$.

topology on the outcomes of neurogenesis-driven network reorganization.

Considerable evidence suggests that the migration and integration of the new cells is promoted by firing activity throughout their development in many ways. Hippocampal neuroblasts arise from progenitor cells in the subgranular zone of the DG and migrate a short distance into the granule layer [3, 10, 24, 25]. Excitatory stimulus in the environment increases the rate of production of new cells [13]. Activity from synaptic inputs from GABAergic interneurons promotes the development of neural processes [16, 32]. Studies have found new granule cells to be preferentially activated by stimulation [37] and to have sensitive synaptic plasticity [40]. Survival of new cells is also increased by integrated activity [26]. Taken together, new cells appear to not only require activity to mature, but also arrange their connections to seek activity. Despite this activity seeking, however, the activity remains globally unchanged.

An epileptogenic injury can significantly alter the neurogenic niche and induce both structural and functional changes in integration (e.g. figure 1). The number of inhibitory cells is reduced by hilar cell death, the size and spread of granule cell synaptic outputs increases due to mossy fiber sprouting and the spatial distribution of granule cells is widened because of granule cell dispersion [9]. Additionally, seizures form a different activity environment in which to mature. The rate of neurogenesis after status epilepticus has been observed to increase [33]. Some neuroblasts migrate ectopically into the hilus and, unlike in the healthy brain $[33,35,39]$, appear to receive synapses from other granule cells [36].

While the pharmacological changes in the local environment evoked by epilepsy have been implicated as effectors of the observed changes [5, 17], the role of the altered network environment itself on new cell incorporation has yet to be thoroughly considered.

In this paper, we develop a minimal computational model of neurogenesis based on the fundamental assumption that new neurons connect and survive within the network in an activitydependent manner. By varying the properties of the original network, we look at the effect of the established network structure on its own reorganization. We find that there is an optimal network topology in our model which is dynamically and structurally robust to the addition of new neurons and leads to focused network adaptation. For other network structures, neuronal integration leads to significant structural and dynamical changes in the network. We thus postulate that there is a range of normal conditions in which neurogenesis could enhance network performance, while in pathological conditions neurogenesis can worsen the pathology instead of being a stable maintenance process.

Our results implicate the structural alteration of the original network in its own structural and functional reorganization. Considerable experimental [11, 15, 44] and computational work $[2,6,12,13,46]$ has focused on the role of neurogenesis in learning. Because of the emphasis on the function of the normal DG, previous theoretical work has not considered the effect of significantly altered initial networks. Computational modeling of the DG and some of the structural changes associated with epilepsy has been done at the network level [14, 28], but not with feedback into the neurogenesisbased network reorganization. Existing theoretical work has focused on either the computational role of neurogenesis or the behavior of static injured networks, but has not considered both aspects as we do here.

\section{Neural integration}

\subsection{Initial network formation}

We focus on the role of the existing network structure on the integration patterns of new cells and the functional and structural network reorganization. In our model, we drastically simplify the cellular and network complexity of the presented simulations. The connectivity structure roughly represents the CA3 layer of the hippocampus [19]. Our network consists of 1000 excitatory cells and a varying number of inhibitory 
(a)

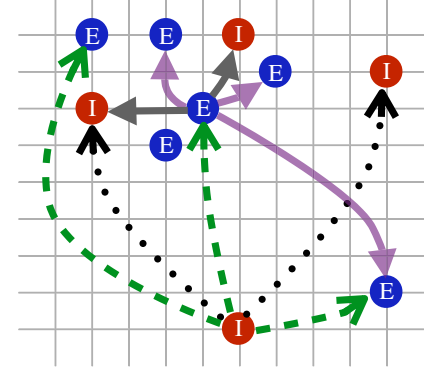

Connection Distance

B $\longrightarrow$ B : $p=0-1$

B $\longrightarrow$ (1) : Local

(1) $\rightarrow$ 1 : Random

(1) - $\rightarrow$ B : Random (b)

Local

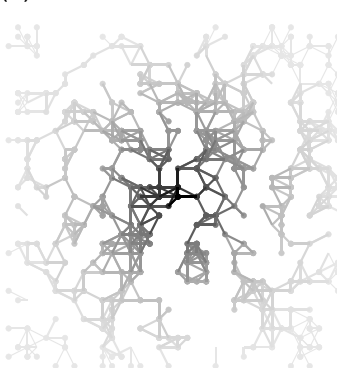

Small-World

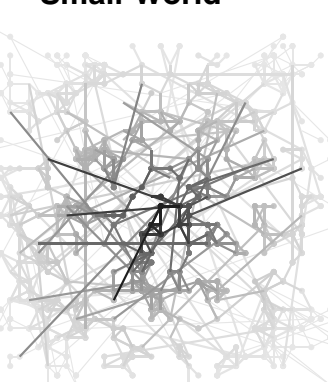

Global

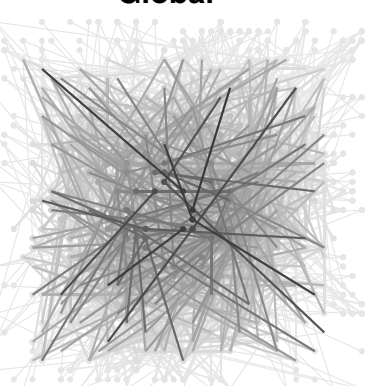

Figure 2. Descriptions of our model network topology. (a) Cartoon schematic of the established network formation. Local excitatory-to-excitatory connections are rewired to a random excitatory cell with probability $p$. $(b)$ Examples of this process on reduced networks for each of the three regimes we consider in this paper: local $(p=0)$, small-world $(p=0.1)$ and global $(p=1)$. Each image is a subset of an excitatory network of 500 cells with an average out-degree of 3. Edges are colored darker according to how close their parent cell is to the center.

cells. We study two levels of inhibition: the normal condition where the established networks prior to neurogenesis have 200 inhibitory cells, and the low inhibition condition where there are 100 inhibitory neurons, corresponding to significant loss of hilar cells. We specify that each excitatory cell will be connected to, on average, 3.5\% of the other excitatory cells. Excitatory neurons are also connected to a small number of nearby inhibitory neurons. Inhibitory cells send out-connections to, on average, 4 other inhibitory cells and 100 excitatory cells. These targets, both excitatory and inhibitory, are selected purely at random.

For simplicity, the cells are positioned on a twodimensional square lattice with 40 lattice sites per side and periodic boundaries. A lattice site can hold at most one excitatory cell. Inhibitory cells are placed on a site independent of its occupancy status, reflective of the fact that inhibitory cells occupy a separate layer and thus are not constrained by soma packing in the excitatory network.

To vary the connectivity properties of excitatory-toexcitatory networks we apply the small-world framework developed by Watts and Strogatz [45]. This allows us to tune the ratio between short-range and long-range neuronal connections in the excitatory cell layer.

If two cells are close to one another, their local connections should have a significant chance of going to the same targets. We accomplish this by determining the downstream (axonal) connections for each cell by looking within a radius containing twice the desired average number of outconnections and connecting to each with probability $1 / 2$. This approach introduces local heterogeneity and prevents a cell from being connected to all neighbors, reducing the amount of recurrence. Our results are robust to changes to the exact connection probability (see supplementary information available at stacks.iop.org/PhysBio/7/046008/mmedia). For each connection, we rewire it to a randomly chosen excitatory cell anywhere in the network with probability, $p$. For $p=0$, no connections are rewired, and for $p=1$ all connections are sent to random targets. The network structure thus depends strongly on the parameter $p$, which effectively controls the ratio of short-range connections to long-range connections.

The rewiring parameter $p$ allows the connectivity of excitatory-excitatory networks to range from purely local $(p=0)$ to purely random $(p=1)$, while preserving many other aspects of the network such as the degree (i.e. cell connectivity) distribution. Between the local and global extremes is a small-world topology. Small-world networks are characterized by having short average path lengths between pairs of nodes, but a high probability that the neighbors of a given node are connected to one another [30]. A cartoon summarizing the result of this process is provided in figure 2(a). We observe a small-world structure in our excitatory networks around $p=0.05-0.2$ (see supplementary information available at stacks.iop.org/PhysBio/7/046008/mmedia). Examples of the above scheme for local, global and small-world networks are shown in figure $2(b)$.

\subsection{Neuronal dynamics}

We represent network activity with integrate-and-fire dynamics with stochastic spontaneous firing. While integrateand-fire is only a very rough approximation of the dynamics of real neurons, it is sufficient for the purposes of our model, for which connectivity, not detailed dynamics, is paramount. 
In the integrate-and-fire scheme, the $k$ th cell has a voltage $V_{k}$ which obeys:

$$
\frac{\mathrm{d} V_{k}}{\mathrm{~d} t}=\left(I_{e / i}+E_{k}-\alpha_{k} V_{k}\right)+\sum_{j} w_{j k} A_{j k} I_{\text {syn }}^{j}\left(t+\tau_{\text {delay }}\right),
$$

where $I_{e / i}$ is the global excitability of excitatory/inhibitory neurons, depending on what class of neuron $k$ belongs to. The initial potentials are distributed uniformly at random between 0 and 1 . The cellular and network parameters are adopted from [19]. We use $I_{i}=0.6$ and $I_{e}=0.73$. The external stimulus is denoted by $E_{k}$, which is 0 for unstimulated cells and 0.4 for those which receive external stimulus. Stimulus, simulating an excitatory input to the DG from the entorhinal cortex [4], occurs at the five centermost excitatory cells, a region chosen to be small enough to not drive bursting across the whole network. The membrane leak constant is $\alpha_{k}$, drawn for each cell from a uniform distribution between 1 and 1.3. The network adjacency matrix elements are denoted by $A_{j k}$, which is 1 if neuron $k$ sends an output to neuron $j$ and 0 otherwise. The synaptic weight is denoted by $w_{j} k$. Synaptic weight is based only on the class of the neurons involved, with $w_{e e}=0.2, w_{e i}=0.4, w_{i e}=-0.4$ and $w_{i i}=-0.7$. The negative sign represents inhibition. Synaptic weight and excitability parameters are inspired by [19], where they were tuned to give controlled dynamics. Finally, the synaptic current from the $j$ th neuron is $I_{\text {syn }}^{j}(t)$, where $I_{\text {syn }}(t)$ comes from a double exponential based on the time $t$ since neuron $j$ fired plus a signaling delay $\tau_{\text {delay }}=0.08 \mathrm{~ms}$ :

$$
I_{\text {syn }}(t)=\mathrm{e}^{-t / \tau_{S}}-\mathrm{e}^{-t / \tau_{F}},
$$

where $\tau_{S}=3 \mathrm{~ms}$ and $\tau_{F}=0.3 \mathrm{~ms}$. This current form is taken from [29].

The voltage dynamics is numerically solved using the Euler method. Membrane potentials are capped below by 0 and if $V_{j}>1$, the neuron $j$ fires. All cells also have a probability of 0.0003 per ms of spontaneously firing, providing a mean background firing rate per cell of $0.3 \mathrm{~Hz}$. This is near the observed spontaneous firing rate for dentate granule cells in rats [20], and our results are robust to variation (supplementary information available at stacks.iop.org/PhysBio/7/046008/mmedia). When a neuron fires, its potential is reset and held at 0 for a refractory time of $8 \mathrm{~ms}$.

\subsection{Network reorganization}

The intent of our simulations is to track the distribution of survivorship and wiring patterns of the new neurons within the existing network based only on activity-dependent integration rules. The rules for this process are independent of the established network structure. We introduce new neurons after the network has settled into steady state dynamics.

A new neuron is introduced every $350 \mathrm{~ms}$ after an initial $1000 \mathrm{~ms}$ of simulation time. The new cell is placed on a randomly chosen unoccupied lattice site. We then proceed to form inward (dendritic) and outward (axonal) connections. We form inward connections by compiling a list of all inward connections of neighboring cells (within radius $R / 2$ ) to the newly added one. This list denotes the possible in-connections. Inputs are drawn from this list by assigning a score $g_{i}$ to each neuron on the list based on the firing rate $f_{i}$ and a small amount of random jitter $\omega$ drawn uniformly between 0 and 1 :

$$
g_{i}=a \frac{f_{i}}{\max _{\{i\}} f_{i}}+(1-a) \omega .
$$

The parameter $a$ determines the amount of randomness in the selection process. We use $a=0.8$ to wash out small differences in firing activity and preserve large ones. The neurons with the highest values of $g$ are selected to be inputs to the new cell, until the appropriate number of inputs is reached. Our results are not sensitive to modest changes in $a$ (supplementary information available at stacks.iop.org/PhysBio/7/046008/mmedia). The motivation for this approach is that the axons that enervate the cells close to a new neuron would also be available to the new dendritic arbor, irrespective of the distance of the respective cell body. Additionally, this approach gives a purely local rule for new cells added to any network.

Similarly, the output connections are drawn from downstream targets of nearby cells. Connections are made to a random subset of possible outputs until the new cell has the same average number of connections as the original network. As before, this is motivated by the assumption of a spatial relationship between the dendrites accessible to a new cell.

In accordance with the high plasticity observed for immature granule cells, during the initial stages of its dynamics, an introduced cell can undergo rapid changes in its connectivity. At intervals of $200 \mathrm{~ms}$ of simulation time, all output connections which do not result in a sufficient number of coincident firing events (defined as a post-synaptic cell firing within $10 \mathrm{~ms}$ after the pre-synaptic cell fires) are broken and new connections are again chosen at random. After $2000 \mathrm{~ms}$ of simulation time, the new cell either dies if its firing rate is less than 100 times the spontaneous background firing rate or matures and ceases to undergo changes in its connections. Our results are robust to the exact value of this threshold (supplementary information available at stacks.iop.org/PhysBio/7/046008/mmedia). When a new cell survives, the total population is kept constant by killing a randomly chosen mature cell.

Birth and maturation rates are chosen so that there is little interaction between two immature cells, to our knowledge a largely undescribed process, and the network activity is well sampled. Spatial activity distributions were not observed to change considerably for the parameters used here, except in response to new cells. Network activity can thus be well measured by $2000 \mathrm{~ms}$ of activity, even though it is not a physiologically relevant time period.

Except where noted, simulations are performed by first running dynamics on the stimulated initial network to establish baseline activity. We then add 500 new cells as described above, and stop the simulation after the last new cell has had an opportunity to mature. Each data point represents 50 realizations. All simulations and data analysis were performed in Matlab 7.7.0 (Mathworks). 


\subsection{Metrics of network activity}

To quantify network-level observations in both structure and dynamics, we employed several metrics, some pre-existing and some new. We describe them here.

\subsubsection{Spatial measurements. To measure and visualize the} firing activity, survivorship and number of reconnection events as a function of location, we break the space into a $20 \times 20$ grid of squares covering the network space, so that each square represents four lattice sites. Activity and reconnection events are both measured as the average value among all cells that fall within a given square. For survivorship, we report the average fraction of new cells that survive within a given square. Correlations between these are found by treating the grid of measurements as a vector and calculating the normalized covariance in the standard way.

2.4.2. Radial component. To measure the mean radial component of output connections, we first create a vector for each new cell that represents the average direction of all outputs. This is defined as

$$
\vec{v}_{i}=\frac{1}{k_{i}} \sum_{j} A_{j i} \frac{\vec{x}_{j}-\vec{x}_{i}}{\left|\vec{x}_{j}-\vec{x}_{i}\right|},
$$

where $k_{i}=\sum_{j} A_{j i}$ is the number of out-connections from the neuron $i$. We then measure the inward radial component by taking the dot product of this mean direction with the inwardpointing unit radial vector $\hat{r}$ at the location of the $i$ th neuron and normalizing by the magnitude of $\vec{v}_{i}: \hat{r} \cdot \vec{v}_{i} /\left|\vec{v}_{i}\right|$. This is averaged for all surviving new neurons in each simulation. Values close to 1 indicate highly radial directionality among new neurons' outputs. Values near zero are consistent with random directions.

2.4.3. Synchronous bursting. We use an existing measure of synchronicity of bursting based on interspike time differences [43]. For an ordered list of all spike times $t_{v}$ for all excitatory cells, the measure $B$ is defined as

$$
B=\frac{1}{\sqrt{N}}\left(\frac{\sqrt{\left\langle\tau_{\nu}^{2}\right\rangle-\left\langle\tau_{\nu}\right\rangle^{2}}}{\left\langle\tau_{\nu}\right\rangle}-1\right),
$$

where $\tau_{v}=t_{v}-t_{v+1}$ is the time difference between subsequent firing events on the excitatory network and angle brackets indicate averages over all such time differences. For large $N$ and all neurons firing as independent Poisson processes, $B=0$, and for synchronous bursting $B=1$.

2.4.4. Spike order. The relative intraburst order of new and established cells is measured as the time difference between the onset of new cells bursting and established cells bursting. We define the onset of a burst as the time at which the number of new or established cells firing simultaneously increases past 4. We make a list of the onset of all new and established cell bursts. For each burst of new cells, we record the time difference to the closest established cell burst onset. The convention is chosen such that an established cell burst that leads a new burst has a negative time difference.

\section{Results}

We find that integration patterns critically depend on the existing network topology, and under normal inhibition fall into one of three categories: local networks for very low $p$, small-world networks for $p \approx 0.1-0.3$ and global networks for, roughly, $p>0.3$. A typical example of each is shown in figure 3 . It provides a template for understanding the details of the results that follow.

For local networks, the spatial distribution of new cells forms an extended region around the stimulus. This region follows a similar distribution as activity, wherein cells in the center participate in bursts that propagate radially outward. Because a cell's location and its firing time are tightly related, cells exhibit some synchrony with much of the active region, but this does not necessarily span the furthest extents. Since axonal connections are selected by their ability to induce downstream firing events, the output structure tends to point into the active region, but not necessarily with a particular focus.

Networks with a high rewiring probability show no localization of survivorship at all, even though a significant fraction, $(1-p)$, of the edges are still local in nature. New cells spread evenly throughout space, resulting in bursts that spread without spatiotemporal order, but with very high synchrony everywhere in space. High synchrony and uniform activity create a distribution of downstream connections that are spread throughout space and highly unfocused.

One might guess that small-world networks should act like slightly more global versions of local networks, but this does not occur. Previous investigations have shown that the small fraction of long-range connections present in smallworld networks can limit the scope of activity by interfering with the ability for the propagation of waves of activity $[29,38]$. Survivorship for small-world networks is localized, and tends to be more focused about the stimulus region. Bursts still tend to propagate in a basically radial fashion, but do not extend as far. This causes the region with highly synchronous firing to include the extent of the active region, since it does not take much time for signals to traverse it. As a result, the axonal connections that can induce firing events are highly oriented toward the active region. Altogether, these integration patterns make the small-world networks respond to stimulus in a more orderly and focused fashion than either the purely local or more global cases.

\subsection{Activity and incorporation patterns of the network in response to external stimulus}

We first consider in detail how the network activity patterns respond to external stimulus for different network topologies. We stimulated five excitatory neurons positioned in the center of the network by applying an additional constant current. Examples of typical dynamics after new cells are added can be found in the supplementary information available at stacks.iop.org/PhysBio/7/046008/mmedia. As expected from the example cases, for established networks with $p<0.2$ and normal inhibition, we see highly localized activation patterns 


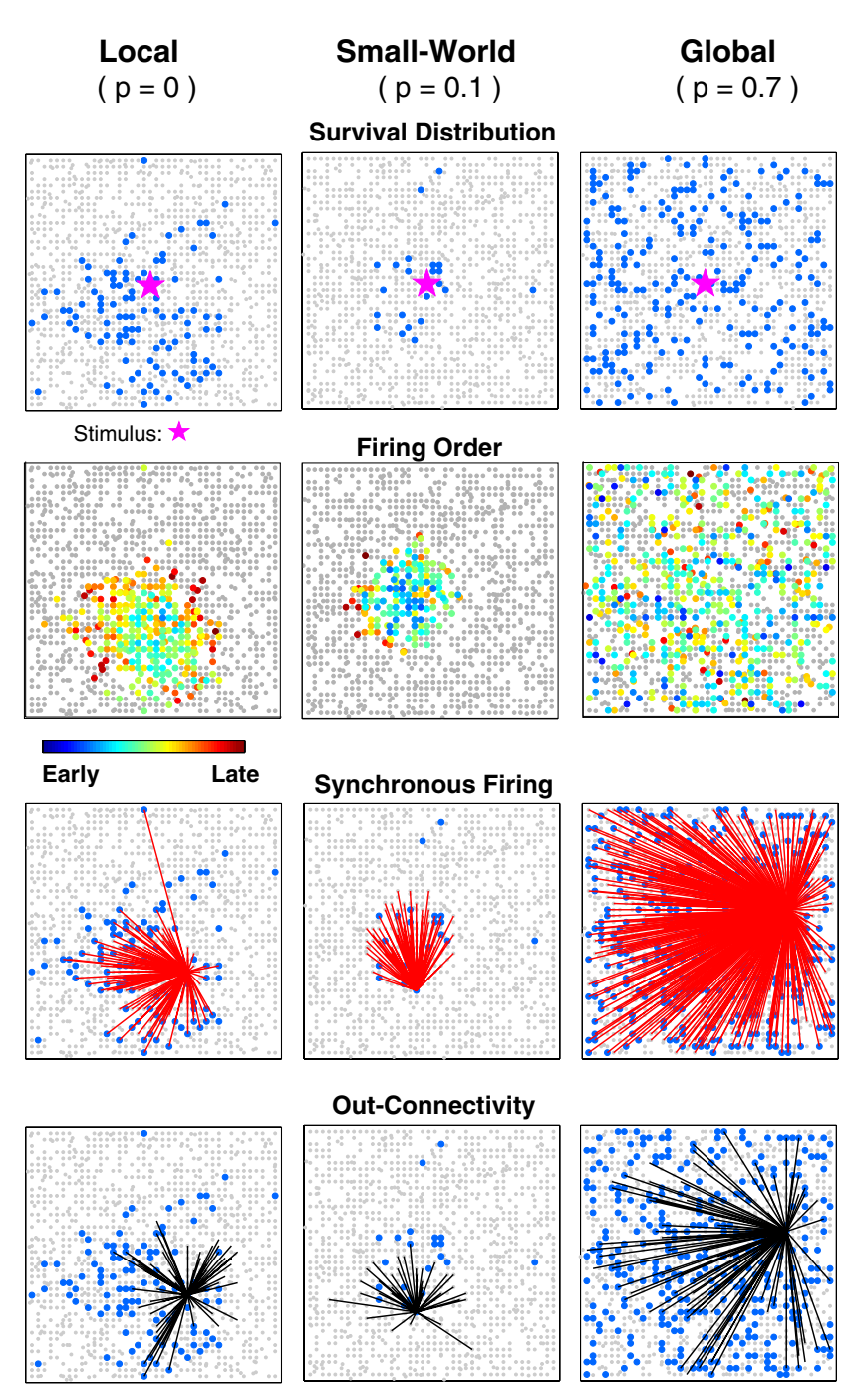

Figure 3. Examples of typical survival patterns of new cells under normal inhibition for each of the three regimes: local $(p=0)$, small-world $(p=0.1)$ and global ( $p=0.7)$. The top row depicts new cells (blue) amidst established cells (gray) and the stimulation point (magenta star). Incorporation is much more concentrated in the small-world case than either local or global. The second row depicts the mean firing delay from burst onset (early to late represented by blue to red). Local and small-world networks exhibit firing that propagates outward from the stimulus, whereas global networks lack spatial order. The third row shows connections from the same cell to cells, new or established, which have highly phase locked firing (red lines). We determine this here by those pairs with a mean phase coherence (as defined in [8]) greater than 0.5. As before, the local and small-world networks are spatially constrained, but the small-world does not expand as far as local networks. Global networks, by contrast, show widespread synchronization. The fourth row highlights the out-connections (black lines) from one new cell. Local topologies, while localized, have less radial orientation of out-connections than small-world topologies, while global networks are highly random. Raster plots from each example can be found in the supplementary information available at stacks.iop.org/PhysBio/7/046008/mmedia.

(figure 4(a), note log color scale), while global connectivity patterns and/or diminished inhibition produce widespread firing. Considering only the changes in the firing rate (figure $4(b)$ ), new cells create an increase in the firing rate that remains near the stimulated region for normal inhibition and low $p$. This confirms that our neurogenesis process can sharpen the response of our network to a stimulus. Increasing the rewiring and decreasing inhibition each result in a global increase of firing rate, with amounts varying depending on the details of the network.

The difference in activity distribution between local and small-world integration is subtle, but very important. The incorporation of new cells into local networks causes much larger changes to the activity profile than in the small-world case (figure $4(c)$ ). The spatial distribution of activity is more stable in $p=0.1$ small-world networks than in purely local networks. The ease with which waves of activity spread through local networks allows them to show more changes in the distribution of activity as new cells are added. Of course, the changes in global networks are larger than both, as activity becomes widespread and uniform throughout space.

The focused activity for small-world networks is also reflected in the spatial distribution of survivorship. Smallworld networks show surviving new cells to be closest to the stimulus in the small-world case (figure 5(a)). To maintain specific activity after incorporation of new cells, as we expect in normal hippocampal conditions, the established network must have relatively local connectivity of the original network and sufficiently high levels of inhibition. Smallworld networks exhibit the highest correlation between initial activity and the spatial distribution of surviving cells, slightly better than purely local networks and much better than more global networks (figure 5(b)). As might be expected since new cells enhance network activity, such small-world networks also show the highest spatial correlation between initial frequency and the chance in frequency (figure $5(c)$ ).

While we observe that under normal inhibition, the smallest number of added cells survive when the network is in the small-world regime, under low inhibition the situation changes dramatically. The spread of new cells is not hindered in any case, allowing the network activity to be uniformly very high after cells integrate (figure $4(a)$ ). Interestingly, with low inhibition, the once-focused small-world regime is associated with the widest distribution of new cells and the worst correlation of network response with initial activity (figures 5(a)-(c)).

\subsection{Innervation patterns of incorporated neurons}

Another important feature of neurogenesis is the innervation pattern of the new cells. We consider each synaptic connection to be spanned by a long axon and short dendrites, such that the direction of a connection is roughly that of the innervating axon. For low values of $p$, a clear pattern of radially oriented outputs toward the stimulation site is observed for introduced cells, as in the examples in figure 3 (see supplementary information available at stacks.iop.org/PhysBio/7/046008/mmedia for the averaged case). This disappears for large $p$.

To quantify this, we measured the average radial component for the output directions of all surviving new cells (figure $5(d)$ ). Networks in the small-world regime show 
Normal Inhibition

(a)

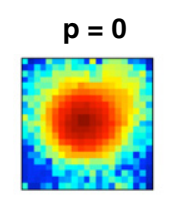

(b)
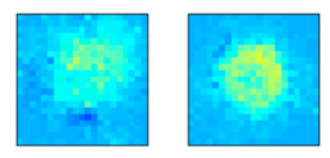

(c)

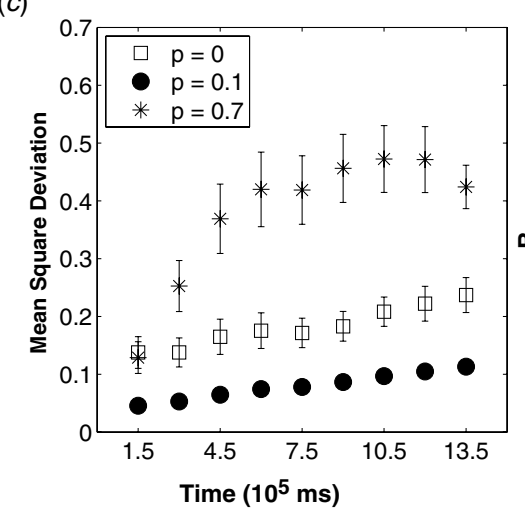

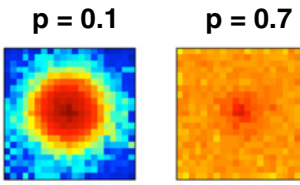

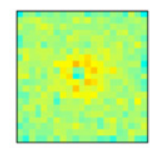

$(d)$
Low Inhibition
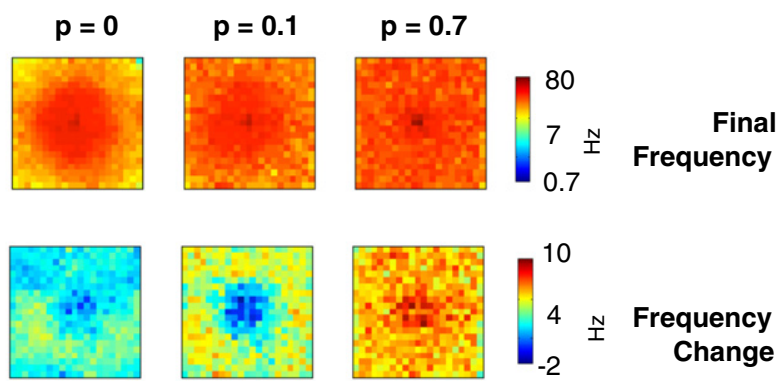

$(e)$
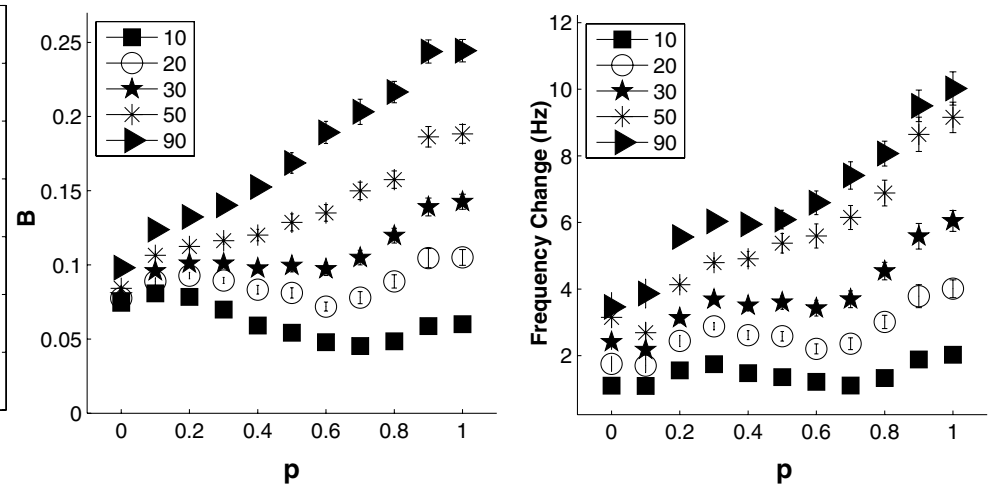

Figure 4. Measures of the distribution of activity. (a) Average spatial distribution of final firing rate (top, $\log$ scale) and (b) difference between final and initial firing rate (bottom, linear scale) for several values of rewiring probability and inhibition. Only normal inhibition and local and small-world networks have spatially constrained final activity and activity change, with small-world having more focused increase of firing rate around the stimulated region. $(c)$ Root mean square change from the initial radial activity profile as a function of simulation time, normalized by the initial maximum firing rate. Higher values indicate a greater change in the spatial distribution of activity. The $p=0.1$ small-world case shows more stable activity distribution than either more local or more global networks. $(d)$ Mean network frequency as a function of the number of cells survived (different markers) and incorporated into the network for normal inhibition. (e) Burst synchrony B, ranging from 0 for Poisson random firing to 1 for exactly simultaneous, as a function of the number of cells survived. Notably, global networks are not functionally robust to the addition of activity-seeking cells. Where present, the error bars denote standard error.

higher radial ordering than those with either more purely local or global connection structure. Local networks, because of their extended active region both initially and after new cell introduction, allow new cells to be driven by and drive activity distant from the initial stimulus. This illustrates yet another way in which activity in the established network is best able to specifically dictate the behavior of new cells in small-world networks. Additionally, low inhibition networks are not able to develop the same degree of directionality as normal networks.

\subsection{Firing dynamics}

Inspired by the spatio-temporal ordering (or lack thereof) of firing events in figure 3 , we consider differences in the firing patterns of new and established cells in reorganized networks. If we look at time traces of the instantaneous firing rate, we see that for small-world networks the activation of new neurons overlaps with that of the already existing cells (figure 5(e)) without a significant lead-lag pattern emerging between the two populations. For random networks, bursts from new cells tend to lead those of the original ones.

To quantify this observation, we measure the time difference between the onset of bursts of the old and new subpopulations (figure $5(f)$ ). This time difference is negative when old cells lead and positive when new cells lead. We restrict ourselves to data taken from the end of the simulation. We see that, in most cases, new cells will lead bursts in the established network. The only case where the established network consistently leads bursts is for low rewiring and normal inhibition. The transition between which cell population leads occurs when the network is in the smallworld regime.

Notably, for low inhibition there is never a regime with a negative time difference. However, the new cell lead time is generally smaller for low inhibition because the established network requires less activity to be driven.

\subsection{Robustness of network activity}

Finally, we wanted to investigate if the observed changes in network dynamics were merely due to different numbers of new cells surviving in the different networks. To address this, we ran simulations that terminated when 100 new cells matured and were successfully incorporated into the network, instead of 500 being introduced to the network, but with many dying. Because of this, some simulations were run for longer time period than others. We quantified the activity patterns with the mean frequency of the network and a measure of synchronous bursting, denoted $B$, taken from [43]. 

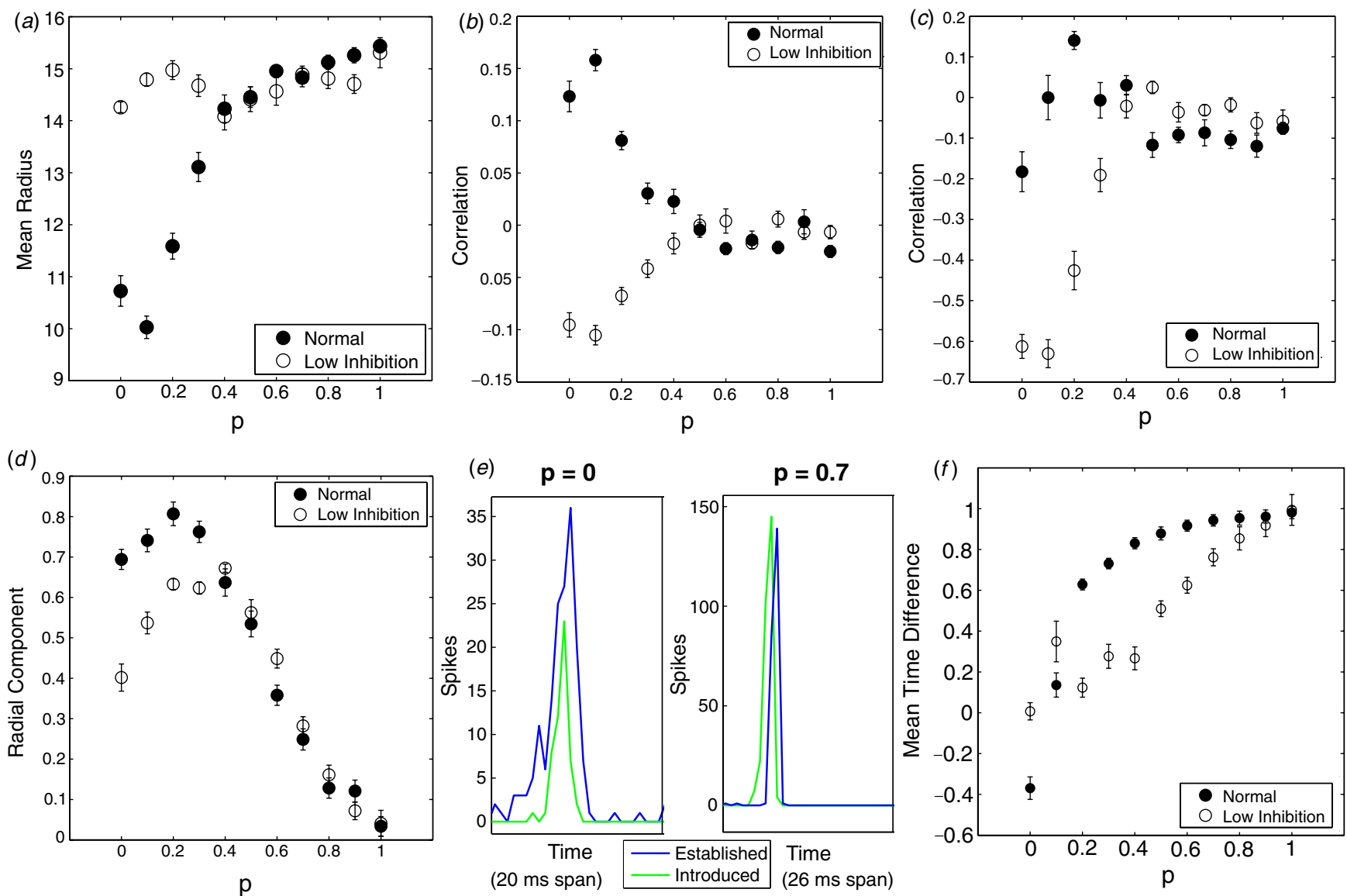

Figure 5. Measures of new cell incorporation and dynamics. (a) The mean radius of surviving cells, defined as the distance from the cell to the center of the stimulus point. (b) Spatial correlation of initial network activity and survival probability. Note that the optimum occurs for $p=0.1$, with neither local nor global networks performing as well. (c) Correlation of initial activity with change in activity. Again, small-world networks are the only ones to show consistent enhancement of initially active regions. Note that the correlation between activity and change in activity may be artificially low, since the locations that have the highest activity may be unable to fire faster due to the refractory time setting a maximum frequency. $(d)$ Mean radial (relative to stimulus location) orientation of new cell out-connections. Small-world networks show the most radial order. $(e)$ Examples of the intraburst structure of instantaneous firing rate for local and global networks. In the local case, new cells fire as part of the burst, whereas in the global case new cells form a population that fires before the established network cells burst. $(f)$ The mean delay in intraburst firing onset of old cells compared to new cells. Local and small-world networks at normal inhibition do not see a large separation of new and old populations. The error bars in all cases denote standard error.

Local and small-world networks are much more robust in terms of the mean firing rate and mean burst synchrony to activity-dependent reorganization than those with more random connections (figures $4(d)$ and $(e)$ ). In all cases, established networks with low $p$ tolerate the incorporation of activity-seeking neurons with only modest increases in activity and synchronous firing. Networks with more global connections start with slightly less activity, but are not able to accommodate the same number of neurons without having increasing firing rate and spontaneous bursting. Decreasing inhibition does not qualitatively change this behavior, although the mean firing rate is generally higher (see supplementary information available at stacks.iop.org/PhysBio/7/046008/mmedia).

\section{Discussion}

In this work, we considered how a network's topology affects its ability to be structurally and functionally maintained by continual addition of new network nodes during neurogenesis. To that end, we constructed a model with very general rules of activity-promoted incorporation of new neurons, and investigated outcomes as a function of the established network environment.

We found that with normal inhibition levels, our networks have stable and focused reorganization by neurogenesis for a relatively narrow range of rewiring probabilities near $p=0.1$, corresponding to the small-world regime of the excitatory network. For networks with that topology, the network activity most precisely dictates the survival location and structural integration of the new cells and the resulting change in the firing rate; yet the network is still able to retain overall dynamical properties. This coincides interestingly with results of others that indicate that many parts of the brain are wired as small-world networks, as well as quantitative estimates of the DG connectivity that imply that it may be a small-world network [14], as well as the suggestion that a small-world DG could provide computational benefits [27]. However, when our network starts in a different topology or has insufficient 
inhibition, the integration patterns are significantly different. Networks that are too local can allow new cells to propagate activity (and thus future new cells) far from the stimulus. Networks that are too global or have low inhibition see a uniform rise in activity throughout space and widespread, nonspecific integration of new cells.

Experimentally observed neurogenic outcomes are normally strongly ordered, but can become far less focused after injury. A variety of experiments show that cells born in an epileptic environment integrate into the network in a more disorganized and less specific fashion than in healthy conditions. Widening of the granule cell layer and ectopic integration of neuroblasts into the dentate hilus are examples of wide dispersal in epilepsy [35]. While cells migrate abnormally due to changes in motility cues [17], how they alter the network dynamics is still an open question. The orientation of these new cells is highly random, in considerable contrast to the ordered picture seen in healthy granule cells. Additionally, neurogenesis accelerates after seizures in terms of both increased proliferation [7, 33] and faster functional integration [31]. Experimental observations have also found that experimental epilepsy reduces learning ability [18, 21, 42], suggesting an altered functional response to stimulus.

We have modeled a portion of the structural network changes observed in human and experimental temporal lobe epilepsy by implementing reduced numbers of inhibitory cells and changes in the network topology. In our model, networks which deviate from the small-world and normal inhibition regime, undergo significant structural and dynamical modifications during incorporation and tend to develop toward higher activity levels, global synchrony and a disordered response to the stimulus. Increasing rewiring induces much greater shifts away from stimulus specificity, with the additional characteristics of activity spreading widely through the network and activity-survival correlation becoming zero. The result is similar to the randomly oriented post-seizure neurons shown in figure 1. Decreasing inhibition also causes even low values of rewiring to result in widespread activity and survival probability, as well as the increased survival rates observed experimentally.

Experiments on rats found that suppressing neurogenesis after status epilepticus reduced the duration and frequency of occurrence of recurrent seizures [22, 23], consistent with the picture that neurogenesis can reinforce and spread epileptic dynamics. In both cases the probability of developing seizures after SE was also reduced. Taken together, it suggests that some injuries that did not induce seizures created an environment that was pushed toward pathological dynamics by neurogenesis. In our model, pushing the network out of the small-world regime, either by making it more local or more global, results in a network less stable to reorganization by neurogenesis, while decreasing inhibition can immediately change dynamics.

Our results suggest that under pathological conditions the newly incorporated cells can exhibit shifts in their activity patterns compared to neurons in the established network. We did not observe these differences under normal conditions. This is possibly related to the observation that hubs (nodes with many more connections than average) can induce epileptic dynamics [28]. The activity-seeking nature of new cells is a mechanism that can create these hub cells. The relative dynamics of newly added neurons into pathological and normal networks would be an interesting avenue for experimental study to gain insight into the possible epileptogenic nature of neurogenesis.

It is important to note that our model is highly abstracted from the anatomical network. The biological DG is host to many neuronal classes, each with distinct morphologies and spatial organization [4]. By far the most numerous are granule cells. They exhibit recurrent synapses only in epileptic conditions, otherwise only targeting other cell types and sending output to the CA3 region of the hippocampus. Excitatory connections between granule cells are provided by mossy cells, which also enervate inhibitory interneurons. The variety of interneuron classes differ greatly in targets and spatial extent, but include both longer and shorter range connections.

Our model does not attempt to include the feature that granule cells interact with one another only through a network of intermediaries, nor does it contain the diversity of inhibitory cell types. Inclusion of these qualities would distinguish functionally between short-range and long-range excitatory connections and introduce much more complicated patterns of inhibitory firing. Because of this, we do not suggest that there is a particular value of $p$ for which our model reproduces the dynamics and structure of either the healthy or epileptic DG. Instead, we emphasize that a change to the balanced excitation and inhibition of a stable network, e.g. adding more highly clustered local connections or spreading excitation widely by random connections, can dramatically alter the impact of adding new cells to it.

Additionally, we choose generic cellular incorporation rules that achieve observed outcomes so our results will not depend strongly on the microscopic details of our model, but rather on the assumptions about the consequences of how cells can act. This is especially important for neurogenesis, as many details of environmental-development interactions are not well understood. The two crucial assumptions of our model are that new cells are promoted by existing activity and can see the local network. Even this simple framework recapitulates many of the important general features of models with more biological detail. In healthy conditions, new cells incorporate into functional units that fire given specific stimulus, as in the detailed learning model of [2]. By looking at the frequency of our networks with no new cells in figure $4(d)$, we see a trend toward somewhat higher base activity for more local networks, made much higher by also decreasing inhibition. Similar behavior was reported in the very detailed and biologically driven hippocampal model of [14].

Our primary result is a general one, describing neurogenesis as a network maintenance process in which functional activity feeds back into structural modification. A neurogenic process that is sufficiently activity dependent to enhance functional clusters can have a complementary topology under which its effect is stable and focused. When the same process operates on networks deviating from this 
regime, however, the network can be driven toward even more pathological behavior. This happens by activity becoming non-localized in the high rewiring case or in the local case by new cells significantly altering the initial activity distribution. Critically, the differences in outcome can be entirely due to differences in the initial neurogenic environment, even without any changes in the underlying biochemistry of the neurogenic process.

\section{Acknowledgments}

CMS would like to acknowledge useful conversations with Sarah Feldt. This work was supported by funding from the University of Michigan Center for Computational Medicine and Biology, National Science Foundation grant DMS0554487 (LS), National Institute of Biomedical Imaging and Bioengineering grant EB008163 (MZ), National Institute of Neurological Disorders and Stroke grant NS058585 (JP) and support from the Tauber Family Foundations and the MaguyGlass Chair in Physics of Complex Systems at Tel Aviv University (EB-J).

\section{References}

[1] Aimone J B, Wiles J and Gage F H 2006 Potential role for adult neurogenesis in the encoding of time in new memories Nat. Neurosci. 9 723-7

[2] Aimone J B, Wiles J and Gage F H 2009 Computational influence of adult neurogenesis on memory encoding Neuron 61 187-202

[3] Altman J and Das G D 1965 Autoradiographic and histological evidence of postnatal hippocampal neurogenesis in rats J. Comp. Neurol. 124 319-35

[4] Amaral D G, Scharfman H E and Lavenex P 2007 The dentate gyrus: fundamental neuroanatomical organization (dentate gyrus for dummies) Prog. Brain Res. 163 3-2

[5] Balu D T and Lucki I 2009 Adult hippocampal neurogenesis: regulation, functional implications, and contribution to disease pathology Neurosci. Biobehav. Rev. 33 232-52

[6] Becker S 2005 A computational principle for hippocampal learning and neurogenesis Hippocampus 15 722-38

[7] Bengzon J et al 1997 Apoptosis and proliferation of dentate gyrus neurons after single and intermittent limbic seizures Proc. Natl Acad. Sci. USA 94 10432-7

[8] Bogaard A, Parent J M, Zochowski M and Booth V 2009 Interaction of cellular and network mechanisms in spatiotemporal pattern formation in neuronal networks J. Neurosci. 29 1677-87

[9] Buckmaster P S and Dudek F E 1997 Network properties of the dentate gyrus in epileptic rats with hilar neuron loss and granule cell axon reorganization J. Neurophysiol. 77 2685-96

[10] Cameron H A, Woolley C S, McEwen B S and Gould E 1993 Differentiation of newly born neurons and glia in the dentate gyrus of the adult rat Neuroscience 56 337-44

[11] Cao L et al 2004 VEGF links hippocampal activity with neurogenesis, learning and memory Nat. Genet. 36 827-35

[12] Chambers R A, Potenza M N, Hoffman R E and Miranker W 2004 Simulated apoptosis/neurogenesis regulates learning and memory capabilities of adaptive neural networks Neuropsychopharmacology 29 747-58

[13] Deisseroth K et al 2004 Excitation-neurogenesis coupling in adult neural stem/progenitor cells Neuron 42 535-52
[14] Dyhrfjeld-Johnsen J et al 2007 Topological determinants of epileptogenesis in large-scale structural and functional models of the dentate gyrus derived from experimental data J. Neurophysiol. 97 1566-87

[15] Farioli-Vecchiol S et al 2008 The timing of differentiation of adult hippocampal neurons is crucial for spatial memory PLoS Biol. 6 e246

[16] Ge S, Pradhan D, Ming G and Song H 2007 GABA sets the tempo for activity-dependent adult neurogenesis Trends Neurosci. 30 1-8

[17] Gong C, Wang T, Huang H and Parent J 2007 Reelin regulates neuronal progenitor migration in intact and epileptic hippocampus J. Neurosci. 27 1803-11

[18] Holmes G L 1997 Epilepsy in the developing brain: lessons from the laboratory and clinic Epilepsia 38 12-30

[19] Jablonski P, Poe G R and Zochowski M 2007 Structural network heterogeneities and network dynamics: A possible dynamical mechanism for hippocampal memory reactivation Phys. Rev. E 75011912

[20] Jakubs K, Nanobashvili A, Bonde S and Ekdahl C T 2006 Environment matters: synaptic properties of neurons born in the epileptic adult brain develop to reduce excitability Neuron 52 1047-59

[21] Jessberger S, Nakashima K and Clemenson Jr G 2007 Epigenetic modulation of seizure-induced neurogenesis and cognitive decline J. Neurosci. 27 5967-75

[22] Jung K H et al 2004 Continuous cytosine-b-darabinofuranoside infusion reduces ectopic granule cells in adult rat hippocampus with attenuation of spontaneous recurrent seizures following pilocarpine-induced status epilepticus Eur. J. Neurosci. 19 3219-26

[23] Jung K H et al 2006 Cyclooxygenase-2 inhibitor, celecoxib, inhibits the altered hippocampal neurogenesis with attenuation of spontaneous recurrent seizures following pilocarpine-induced status epilepticus Neurobiol. Dis. $23237-46$

[24] Kaplan M S and Hinds J W 2007 Neurogenesis in the adult rat: electron microscopic analysis of light radioautographs Science 197 1092-4

[25] Kuhn H G, Dickinson-Anson H and Gage F H 1996 Neurogenesis in the dentate gyrus of the adult rat: age-related decrease of neuronal progenitor proliferation J. Neurosci. 16 2027-33

[26] Lin C W et al 2010 Genetically increased cell-intrinsic excitability enhances neuronal integration into adult brain circuits Neuron 65 32-9

[27] Manev R and Manev H 2005 The meaning of mammalian adult neurogenesis and the function of newly added neurons: the 'small-world' network Med. Hypothesis 64 114-7

[28] Morgan R J and Soltesz I 2008 Nonrandom connectivity of the epileptic dentate gyrus predicts a major role for neuronal hubs in seizures Proc. Natl Acad. Sci. USA 105 6179-84

[29] Netoff T, Clewley R, Arno S, Keck T and White J 2004 Epilepsy in small-world networks J. Neurosci. 24 8075-83

[30] Newman M E J 2003 The structure and function of complex networks SIAM Rev. 45 167-256

[31] Overstreet-Wadiche L, Bromberg D and Bensen A 2006 Seizures accelerate functional integration of adult-generated granule cells J. Neurosci. 26 4095-103

[32] Overstreet-Wadiche L S and Westbrook G L 2006 Functional maturation of adult-generated granule cells Hippocampus 16 208-15

[33] Parent J M et al 1997 Dentate granule cell neurogenesis is increased by seizures and contributes to aberrant network reorganization in the adult rat hippocampus J. Neurosci. $173727-38$

[34] Parent J M 2002 The role of seizure-induced neurogenesis in epileptogenesis and brain repair Epilepsy Res. 50 179-89 
[35] Parent J M, Elliott R C, Pleasure R J, Barbaro N M and Lowenstein D H 2006 Aberrant seizure-induced neurogenesis in experimental temporal lobe epilepsy Ann. Neurol. 59 81-91

[36] Pierce J P, Melton J, Punsoni M, McCloskey D P and Scharfman H E 2005 Mossy fibers are the primary source of afferent input to ectopic granule cells that are born after pilocarpine-induced seizures Exp. Neurol. 196 316-31

[37] Ramirez-Amaya V, Marrone D F, Gage F H, Worley P F and Barnes C A 2006 Integration of new neurons into functional neural networks J. Neurosci. 26 12237-41

[38] Roxin A, Riecke H and Solla S A 2004 Self-sustained activity in a small-world network of excitable neurons Phys. Rev. Lett. 92198101

[39] Scharfman H E, Goodman J H and Sollas A L 2000 Granule-like neurons at the hilar/CA3 border after status epilepticus and their synchrony with area CA3 pyramidal cells: functional implications of seizure-induced neurogenesis J. Neurosci. 20 6144-58

[40] Schmidt-Hieber C, Jonas P and Bischofberger J 2004 Enhanced synaptic plasticity in newly generated granule cells of the adult hippocampus Nature 429 184-7
[41] Silva A J, Zhou Y, Rogerson T, Shobe J and Balaji J 2009 Molecular and cellular approaches to memory allocation in neural circuits Science $\mathbf{3 2 6} 391-5$

[42] Stafstrom C E, Chronopoulos A, Thurber S, Thompson J L and Holmes G L 1993 Age-dependent cognitive and behavioral deficits after kainic acid seizures Epilepsia 34 420-32

[43] Tiesinga P H E and Sejnowski T J 2004 Rapid temporal modulation of synchrony by competition in cortical interneuron networks Neural Comput. 16 251-75

[44] Van Praag H, Christie B R, Sejnowski T J and Gage F H 1999 Running enhances neurogenesis, learning, and long-term potentiation in mice Proc. Natl Acad. Sci. USA 96 13427-31

[45] Watts D and Strogatz S 1998 Collective dynamics of 'small-world' networks Nature 393 440-2

[46] Wiskott L, Rasch M J and Kempermann G 2006 A functional hypothesis for adult hippocampal neurogenesis: avoidance of catastrophic interference in the dentate gyrus Hippocampus 16 329-43

[47] Zhao C, Deng W and Gage F 2008 Mechanisms and functional implications of adult neurogenesis Cell 132 645-60 